\title{
Human umbilical cord mesenchymal stem cells alleviate interstitial fibrosis and cardiac dysfunction in a dilated cardiomyopathy rat model by inhibiting TNF- $\alpha$ and TGF-ß1/ERK1/2 signaling pathways
}

\author{
CHANGYI ZHANG ${ }^{1}$, GUICHI ZHOU ${ }^{2}$, YEZENG CHEN ${ }^{2}$, SIZHENG LIU $^{2}$, FEN CHEN $^{3,4}$, \\ LICHUN XIE ${ }^{3,4}$, WEI WANG ${ }^{1}$, YONGGANG ZHANG ${ }^{1}$, TIANYOU WANG ${ }^{4}$, XIULAN LAI $^{2}$ and LIAN MA ${ }^{2,3,5}$ \\ Departments of ${ }^{1}$ Cardiology and ${ }^{2}$ Pediatrics, Second Affiliated Hospital of Shantou University Medical College, Shantou, \\ Guangdong 515041; ${ }^{3}$ Department of Pediatrics, Maternal and Child Health Care Hospital of Pingshan District, Shenzhen, \\ Guangdong 518000; ${ }^{4}$ Department of Pediatrics, Beijing Children's Hospital, Capital Medical Hospital, Beijing 100032; \\ ${ }^{5}$ Department of Pediatrics, Maternal and Child Health Care Hospital of Shenzhen University, \\ Guangdong 518000, P.R. China
}

Received December 27, 2016; Accepted August 18, 2017

DOI: $10.3892 / \mathrm{mmr} .2017 .7882$

\begin{abstract}
Dilated cardiomyopathy (DCM) is a disease of the heart characterized by pathological remodeling, including patchy interstitial fibrosis and degeneration of cardiomyocytes. In the present study, the beneficial role of human umbilical cord-derived mesenchymal stem cells (HuMSCs) derived from Wharton's jelly was evaluated in the myosin-induced rat model of DCM. Male Lewis rats (aged 8-weeks) were injected with porcine myosin to induce DCM. Cultured HuMSCs ( $1 \times 10^{6}$ cells/rat) were intravenously injected 28 days after myosin injection and the effects on myocardial fibrosis and the underlying signaling pathways were investigated and compared with vehicle-injected and negative control rats. Myosin injections in rats (vehicle group and experimental group) for 28 days led to severe fibrosis and significant deterioration of cardiac function indicative of DCM. HuMSC treatment reduced fibrosis as determined by Masson's staining of collagen deposits, as well as quantification of molecular markers of myocardial fibrosis such as collagen I/III, profibrotic factors transforming growth factor- $\beta 1$ (TGF- $\beta 1$ ), tumor necrosis factor- $\alpha(\mathrm{TNF}-\alpha)$, and connective tissue growth
\end{abstract}

Correspondence to: Professor Lian Ma, Department of Pediatrics, Maternal and Child Health Care Hospital of Shenzhen University, 6 South LongXing Road, Shenzhen, Guangdong 518000, P.R. China E-mail: malian8965@sina.com

Dr Xiulan Lai, Department of Pediatrics, Second Affiliated Hospital of Shantou University Medical College, 69 North DongXia Road, Shantou, Guangdong 515041, P.R. China

E-mail: laixiulan7@163.com

Key words: cardiac dysfunction, dilated cardiomyopathy, human umbilical cord mesenchymal stem cells, TNF- $\alpha$, TGF- $\beta 1$ signaling factor (CTGF). HuMSC treatment restored cardiac function as observed using echocardiography. In addition, western blot analysis indicated that HuMSC injections in DCM rats inhibited the expression of TNF- $\alpha$, extracellular-signal regulated kinase $1 / 2$ (ERK1/2) and TGF- $\beta 1$, which is a master switch for inducing myocardial fibrosis. These findings suggested that HuMSC injections attenuated myocardial fibrosis and dysfunction in a rat model of DCM, likely by inhibiting TNF- $\alpha$ and the TGF- $\beta 1 /$ ERK1/2 fibrosis pathways. Therefore, HuMSC treatment may represent a potential therapeutic method for treatment of DCM.

\section{Introduction}

Dilated cardiomyopathy (DCM) is characterized by dilatation of the ventricles, patchy interstitial fibrosis and degenerated cardiomyocytes. Along with genetic abnormalities, myocarditis has been considered to be a major factor that leads to the development of DCM (1). There is evidence for the role of the immune system in the pathogenesis of myocarditis and subsequent development of DCM $(2,3)$. Experimental autoimmune myocarditis (EAM), which mimics human fulminant myocarditis in the acute phase and human DCM in the chronic phase, is induced by immunization of rats with cardiac myosin (4).

DCM is a progressive disorder, and despite available state-of-the art treatment such as diuretic or cardiac resynchronization therapy (CRT), it is characterized by high morbidity and mortality rates (5). Mesenchymal stem cell (MSC) therapy may be a potential novel approach for treatment of cardiovascular injury and for promotion of tissue regeneration (6). However, various stem cell trials for cardiovascular indications have been disappointing, possibly due to of the use of autologous stem cells (7). Cardiovascular disease patients typically belong to the older age groups, where numerous risk factors may compromise stem cell function $(8,9)$. Allogeneic MSCs may be easily scaled and quality-controlled, and are 
immunologically relatively well-tolerated, allowing their use for stem cell trials that has exceed the feasibility of autologous strategies. Therefore, previous studies using allogeneic stem cells have been established, including the Percutaneous Stem Cell Injection Delivery Effects on Neomyogenesis in patients with DCM trial revealed that the rate of major adverse cardiac events was significantly lower in patients treated with allogenic vs. autologous stem cells (10-12). An additional source of MSCs are human umbilical cord-derived mesenchymal stem cells (HuMSCs). They are generally discarded as medical waste after delivery, thus, their use is of little ethical concern. There are two arteries and a vein in the umbilical cord which are surrounded by a hyaluronic acid-rich extracellular matrix (ECM) also termed Wharton's jelly (WJ). MSCs from umbilical cord WJ are easily isolated and cultured in vitro. Additionally, they can be differentiated in vitro into several tissue types (13). There are several distinct advantages for HuMSCs over other MSCs: i) They have low immunogenicity, attributable to low expression of human leukocyte antigen major histocompatibility complex I (MHC I); and ii) they lack MHC II molecules and co-stimulatory antigens, such as CD80 and CD86. Therefore, HuMSCs are regarded as immunologically safe for use in allogeneic clinical therapies $(14,15)$. Previous studies demonstrated that HuMSCs possess many potential advantages for cell-based treatment of diseases, such as systemic lupus erythematosus (16), rheumatoid arthritis (17), diabetes (18) and myocardial ischemia $(19,20)$. However, the potential beneficial effects of HuMSCs on DCM and the underlying signaling events remain speculative and remain to be fully elucidated.

A variety of signal transduction pathways are involved in myocardial fibrogenesis leading to DCM. For example, activation of the ERK/transforming growth factor- $\beta 1$ (TGF- $\beta 1$ ) pathway was associated with upregulated collagen deposition contributing to myocardial fibrosis (21-23).

In the present study, a DCM rat model was established in order to investigate the therapeutic efficiency of HuMSCs in DCM rats and to analyze the potential signaling mechanisms.

\section{Materials and methods}

Animals. Lewis rats (male, 8-weeks old; weight, 180-200 g, $\mathrm{n}=24$ ) were obtained from Vital River Laboratories (Beijing, China) and maintained in an air-conditioned animal facility at Shantou University Medical College (Shantou, China) under $25^{\circ} \mathrm{C}$ and $70 \%$ humidity conditions with a 12-h light/dark cycle. Throughout the experiments for the current study, all animals were treated in accordance with the institutional guidelines for animal experiments. The Animal Care and Use Committee of the Shantou University Medical College approved all experimental procedures.

Preparation of HuMSCs. HuMSCs were prepared as previously described (24). Briefly, human umbilical cords from pregnant women (12 volunteers; age, 25-35 years; recruited from February 2012 to November 2013) who underwent full-term Caesarian sections were collected from the Second Affiliated Hospital of Shantou University Medical College immediately, washed, and cut into 2-3-cm thick sections. Written informed consent was obtained from all participants. After separating the arteries and veins, WJ was sliced into smaller fragments and transferred to $75 \mathrm{~cm}^{2}$ flasks containing Dulbecco's modified Eagle's medium/F12 media (Sigma-Aldrich; EMD Millipore, Billerica, MA, USA) supplemented with $10 \%$ fetal bovine serum (Gibco; Thermo Fisher Scientific, Inc., Waltham, MA, USA), $100 \mu \mathrm{g} / \mathrm{ml}$ penicillin/streptomycin (Shanghai Bioscience, Shanghai, China), $1 \mathrm{~g} / \mathrm{ml}$ amphotericin B (Gilead Sciences, Inc., San Dimas, CA, USA), 5 ng/ml epidermal growth factor (Invitrogen; Thermo Fisher Scientific, Inc.), and $5 \mathrm{ng} / \mathrm{ml}$ basic fibroblast growth factor (Sigma-Aldrich; EMD Millipore). HuMSC were cultured for 5-7 days at $37^{\circ} \mathrm{C}$ in $5 \%$ $\mathrm{CO}_{2}$ to allow migration of cells from the explants. After three passages the cells were used for subsequent experiments. Ethical approval was obtained from the Institutional Review Board of Shantou University Medical College.

Generation of DCM rat model. Lewis rats were injected in the footpads with antigen-adjuvant emulsion in accordance with a procedure described previously (4). Briefly, purified porcine cardiac myosin (Sigma-Aldrich; EMD Millipore) was dissolved in $10 \mathrm{mM}$ PBS and emulsified with an equal volume of complete Freund's adjuvant with $10 \mathrm{mg} / \mathrm{ml}$ Mycobacterium tuberculosis (Sigma-Aldrich; EMD Millipore). On day 0 , rats received a single immunization with a total of $0.2 \mathrm{ml}$ emulsion per rat at two subcutaneous sites (both footpads). At 28 days after immunization, surviving DCM rats $(n=16)$ were divided into two treatment groups: i) $0.2 \mathrm{ml}$ PBS only (vehicle control group, $\mathrm{n}=8$ ), or ii) $0.2 \mathrm{ml}$ HuMSCs $\left(1 \times 10^{6}\right.$ cells/animal; experimental group, $\left.n=8\right)$. HuMSCs or vehicle (PBS) was administered intravenously via the tail vein. Age matched Lewis rats without immunization were used as negative controls (negative control group, $n=8$ ). The echocardiography and myocardial pathological section were used to confirm the success of the DCM rat model (25).

Echocardiographic studies. Two-dimensional echocardiography was performed 56 days after myosin injections under isoflurane anesthesia (1.5-2.0\% volume in air), and using a 13-MHz transducer linked to an ultrasound system (Acuson Antares, Siemens, Healthineers, Erlangen, Germany). M-mode images were used to obtain measurements of the left ventricular end systolic dimension (LVEDs), left ventricular end diastolic dimension (LVEDd), interventricular septal thickness (IVS), left ventricular posterior wall thickness (LVPW) and fractional shortening (FS \%). The average of three beats was used for each parameter. FS (\%) was calculated as follows [(LVEDd - LVEDs)/LVEDd] x100. All echocardiography analysis was performed offline and investigators were blinded to the treatment groups.

Histopathological studies. Following echocardiographic analysis, rats were sacrificed using cervical dislocation 56 days after myosin injection. The hearts were excised and weighed to calculate the heart/body weight $(\mathrm{HW} / \mathrm{BW})$ ratio. The hearts were subsequently fixed in $4 \%$ paraformaldehyde, embedded in paraffin, and sectioned at $4-\mu \mathrm{m}$ thickness. These sections were stained with either hematoxylin and eosin (H\&E) for infiltration of inflammatory cells, or Masson's trichrome stain for collagen fibers. Slides were viewed under a high-power light microscope. The area of myocardial fibrosis (blue color) 
Table I. List of quantitative polymerase chain reaction primers.

\begin{tabular}{llc}
\hline Primer & \multicolumn{1}{c}{ Forward $\left(3^{\prime}-5^{\prime}\right)$} & Reverse $\left(3^{\prime}-5^{\prime}\right)$ \\
\hline Col I & CGTGGAAACCTGATGTATGCT & CCTATGACTTCTGCGTCTGG \\
Col III & GATCCTAACCAAGGCTGCAA & ATCTGTCCACCAGTGCTTCC \\
TGF- $\beta 1$ & ATTCCTGGCGTTACCTTGG & AGCCCTGTATTCCGTCTCCT \\
TNF- $\alpha$ & GCTCCCTCTCATCAGTTCCA & GCTTGGTGGTTTGCTACGAC \\
GAPDH & AGAAGGCTGGGGCTCATTTG & AGGGGCCATCCACAGTCTTC
\end{tabular}

in left ventricular (LV) tissue sections following Masson's staining was quantified using a color image analyzer (Mac Scope; Mitani Co., Fukui, Japan) and measured as the collagen volume fraction $(\mathrm{CVF})=$ (area of the collagen/area of field of vision) $\mathrm{x} 100$. Ten randomly selected sections (magnification, x100) from each rat were analyzed and the results averaged.

Reverse transcription-quantitative polymerase chain reaction $(R T-q P C R)$. Collagen I, III, TGF- $\beta 1$ and tumor necrosis factor- $\alpha$ (TNF- $\alpha$ ) mRNA expression levels in myocardial tissue were detected using RT-qPCR. The total RNA was isolated from $50 \mathrm{mg}$ heart tissue using TRIzol reagent (Thermo Fisher Scientific, Inc.) according to the manufacturer's instructions. The primers were designed and synthesized by Shanghai Sangon Biotech Co., Ltd. (Shanghai, China). The primer sequences are presented in Table I. Primer concentration was $200 \mathrm{pM}$. RNA (500 ng) in a $10 \mu \mathrm{l}$ reaction mixture was reverse transcribed using the PrimeScript RT reagent kit with gDNA Eraser (Perfect Real-Time; Takara Biotechnology Co., Ltd., Dalian, China). The reactions were incubated first at $37^{\circ} \mathrm{C}$ for $15 \mathrm{~min}$, followed by inactivation at $85^{\circ} \mathrm{C}$ for $5 \mathrm{sec}$ and finally held at $4^{\circ} \mathrm{C}$. The qPCR reaction was performed using SYBR Premix Ex Taq ${ }^{\text {TM }}$ II (Tli RNaseH Plus; Takara Biotechnology Co., Ltd.) and detection was performed with the CFX96 ${ }^{\mathrm{TM}}$ PCR detection system (Bio-Rad Laboratories, Inc., Hercules, CA, USA). The reaction cycles were: Denaturation at $95^{\circ} \mathrm{C}$ for $30 \mathrm{sec}$, followed by 40 cycles of denaturation at $95^{\circ} \mathrm{C}$ for $5 \mathrm{sec}$ and annealing and extension at $60^{\circ} \mathrm{C}$ for $30 \mathrm{sec}$. The relative level of gene expression was normalized to the expression of the housekeeping gene GAPDH using the $2^{-\Delta \Delta \mathrm{Cq}}$ method (26).

Western blot analysis. Heart tissues were treated in radioimmunoprecipitation assay lysis buffer $[100 \mathrm{mM} \mathrm{NaCl}, 20 \mathrm{mM}$ Tris (pH 8.0), 1 mM EDTA (pH 8.0), 0.5\% Triton X-100, 0.5\% Nonidet P-40] to extract total protein, which were quantified using bicinchoninic acid method (Beyotime Institute of Biotechnology, Haimen, China). Total protein $(30 \mu \mathrm{g})$ was separated by $10 \%$ SDS-PAGE gel and transferred electrophoretically to polyvinylidene difluoride membranes (EMD Millipore) for western blot analysis. Membranes were blocked with 5\% non-fat dry milk in Tris-buffered saline [20 mM Tris (pH 6.8), $137 \mathrm{mM} \mathrm{NaCl}$ ] with $0.1 \%$ Tween-20, washed, and incubated at $4^{\circ} \mathrm{C}$ for $16 \mathrm{~h}$ with the following primary antibodies: GAPDH (catalog no. D4C6R, 1:10,000), extracellular signal-regulated kinase (ERK)-1/2 (catalog no. 9258, 1:1,000), phosphorylated (p)-ERK-1/2 (catalog no. 4668, 1:2,000), p38 mitogen activated protein kinase (MAPK) (catalog no. 8690, 1:1,000) and p-p38 MAPK (catalog no. 4511, 1:1,000) were all purchased from Cell Signaling Technology, Inc. (Danvers, MA, USA). Collagen III (catalog no. ab7778, 1:5,000) and connective tissue growth factor (CTGF, catalog no. ab6992, 1:1,000) were purchased from Abcam (Cambridge, UK). Membranes were washed and incubated with a 1:2,000 dilution of horseradish peroxidase-labeled goat anti-rabbit IgG secondary antibody (catalog no. 4050-05; Southern Biotechnology Associates, Inc., Birmingham, AL, USA) for $1 \mathrm{~h}$ at room temperature. Immunoreactive protein bands were visualized using the ECL Plus chemiluminescence kit (EMD Millipore). Bands were analyzed using Image Pro Plus 6.0 (Media Cybernetics, Inc., Rockville, MD, USA) and protein expression quantities were determined according to the following calculation: Integrated optical density $(\mathrm{IOD})=$ density (mean) $\mathrm{x}$ area.

Statistical analysis. Data are expressed as mean \pm standard deviation. Analyses of the differences between groups were performed using one-way analysis of variance followed by Tukey's multiple comparison test. $\mathrm{P}<0.05$ was considered statistically significant.

\section{Results}

Effects of HuMSCs on myocardial fibrosis and cardiac function in DCM rats. HuMSCs treatment significantly reduced myocardial fibrosis (Fig. 1A and B). HW/BW was significantly greater in vehicle control rats compared with negative control rats. HuMSC treatment significantly reduced HW/BW when compared with those in vehicle control rats (Fig. 1C, Table II, $\mathrm{P}<0.01$ vs. normal, $\mathrm{P}<0.05$ vs. control). Echocardiographic analyses revealed a significant impairment in systolic and diastolic function (Fig. 1A). DCM rats demonstrated LV remodeling with increased LVEDd, LVEDs and reduced FS in vehicle-treated DCM rats compared with untreated rats (Fig. 1C, Table II, $\mathrm{P}<0.01$ vs. normal), indicating impaired myocardial function. HuMSC treatment significantly reversed these changes (Fig. 1C, Table II, P<0.01 vs. control).

Histopathological examination revealed that the hearts of DCM rats showed severe fibrosis compared with negative control rats (Fig. 1A). Inflammatory cellular infiltration was not observed in the hearts of the three groups as identified by H\&E staining (Fig. 1A). The area of myocardial fibrosis as quantified by Masson's staining of collagen deposits was approaching $30 \%$ in DCM rats (Fig. 1B).

Effects of HuMSCs on molecular markers of myocardial fibrosis in DCM rats. To confirm the positive effect of HuMSCs on myocardial fibrosis, RT-qPCR analysis on molecular 
A
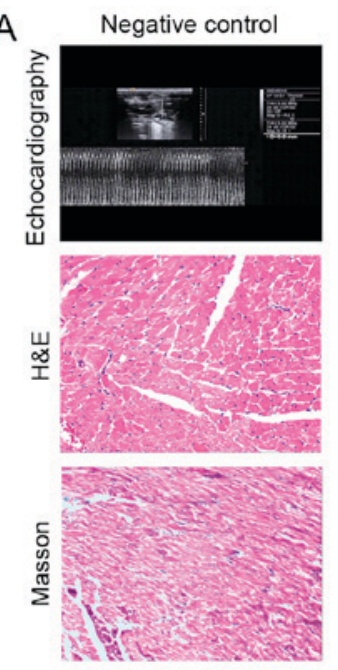

C

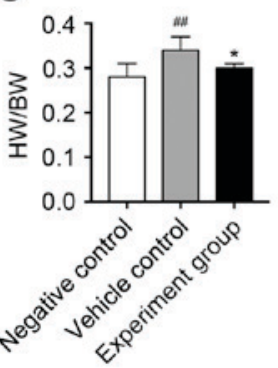

Vehicle control
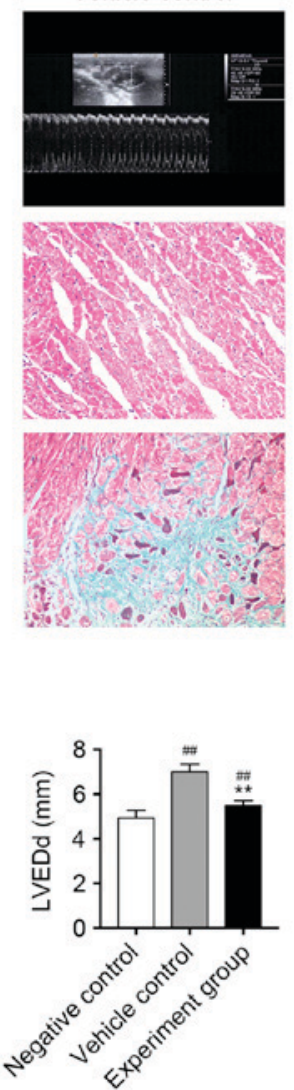

Experiment group
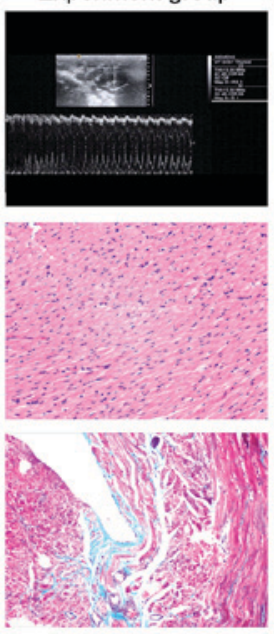

\section{B}
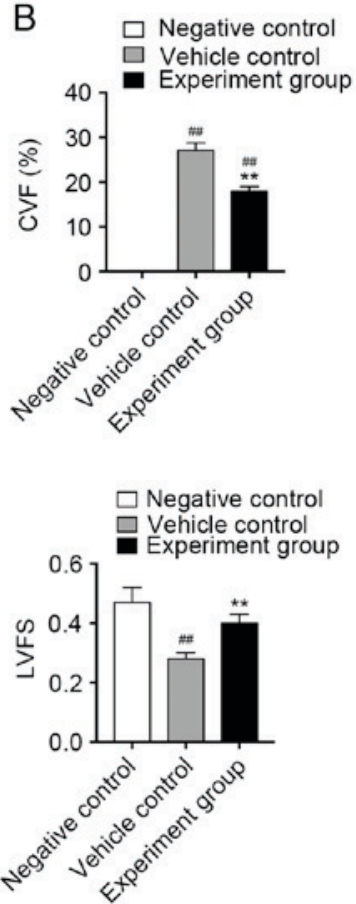

Figure 1. (A) H\&E and Masson's staining in left ventricular tissue slices in age-matched untreated rats, immunized rats treated with vehicle and immunized rats treated with HuMSCs. Magnification, x100. (B) CVF for Masson's staining in left ventricular tissue slices from the different treatment groups. ${ }^{\# \#} \mathrm{P}<0.01$ vs. negative control; ${ }^{*} \mathrm{P}<0.05,{ }^{* *} \mathrm{P}<0.01$ vs. vehicle control, $\mathrm{n}=8$. (C) Heart cavity measurements. ${ }^{\# \#} \mathrm{P}<0.01$ vs. negative control; ${ }^{*} \mathrm{P}<0.05$, ${ }^{* *} \mathrm{P}<0.01$ vs. vehicle control, $\mathrm{n}=8$. H\&E, hematoxylin and eosin; HuMSCs, human umbilical cord-derived mesenchymal stem cells; CVF, collagen volume fraction; HW/BW, heart weight/body weight; LVEDd, left ventricular dimension in end diastole; LVEDs, left ventricular dimension in end systole.

Table II. Echocardiographic parameters 56 days after treatment of DCM rats with HuMSCs in.

\begin{tabular}{lccc}
\hline Characteristic & $\begin{array}{c}\text { Negative } \\
\text { control }\end{array}$ & $\begin{array}{c}\text { Vehicle } \\
\text { control }\end{array}$ & Experiment \\
\hline HW/BW & $0.28 \pm 0.03$ & $0.34 \pm 0.03^{\mathrm{a}}$ & $0.30 \pm 0.01^{\mathrm{b}}$ \\
LVEDd (mm) & $4.93 \pm 0.35$ & $7.00 \pm 0.34^{\mathrm{a}}$ & $5.48 \pm 0.22^{\mathrm{a}, \mathrm{c}}$ \\
LVEDs (mm) & $2.82 \pm 0.86$ & $5.02 \pm 0.31^{\mathrm{a}}$ & $3.02 \pm 0.36^{\mathrm{c}}$ \\
IVS (mm) & $2.16 \pm 0.20$ & $2.12 \pm 0.19$ & $2.10 \pm 0.20$ \\
LVWP (mm) & $2.34 \pm 0.11$ & $2.12 \pm 0.26$ & $2.22 \pm 0.15$ \\
LVFS & $0.47 \pm 0.05$ & $0.28 \pm 0.02^{\mathrm{a}}$ & $0.40 \pm 0.03^{\mathrm{c}}$ \\
\hline
\end{tabular}

a 0.01 vs. normal; ${ }^{b} \mathrm{P}<0.05$ vs. control; ${ }^{c} \mathrm{P}<0.01$ vs. control. HW/BW, heart weight/body weight; LVEDd, left ventricular dimension in end diastole; LVEDs, left ventricular dimension in end systole; IVS, interventricular septal thickness; LVPW, left ventricular posterior wall thickness; LVFS, left ventricular fractional shortening; DCM, dilated cardiomyopathy; Negative control, age-matched untreated rats; Vehicle control, DCM rats treated with vehicle; Experiment, DCM rats treated with HuMSCs.

markers of myocardial fibrosis was performed (Fig. 2). Collagen I, III and the profibrotic factors TGF- $\beta 1$ and
TNF- $\alpha$ were significantly upregulated in vehicle-treated DCM rats, compared with negative control rats (Fig. 2, $\mathrm{P}<0.01)$. By contrast, treatment with HuMSCs significantly reduced mRNA expression of collagen-I, III, TGF- $\beta 1$ and TNF- $\alpha$ when compared with vehicle-treated DCM rats (Fig. 2, P<0.01). At the protein level, CTGF and collagen-III were significantly upregulated in vehicle-treated DCM rats compared with negative control rats (Fig. $3, \mathrm{P}<0.05, \mathrm{P}<0.01$ ). However, treatment with HuMSCs significantly reduced the myocardial protein expression of collagen-III and CTGF (Fig. 3, $\mathrm{P}<0.05, \mathrm{P}<0.01$ ).

Effects of HuMSCs on activation of MAPK signaling in DCM rats. TGF- $\beta 1$ activates numerous non-canonical signaling pathways, including MAPK pathways. Therefore, in order to investigate the signaling mechanisms, which HuMSCs use to reduce myocardial fibrosis in DCM rats, activation of p38-MAPK and ERK1/2 was quantified (Fig. 4). Myocardial protein expression of p38-MAPK, ERK1/2, p-p38-MAPK and $\mathrm{p}$-ERK1/2 were quantified using western blot analysis. P-ERK1/2 levels significantly increased in vehicle control rats compared with negative control rats, whereas p-p38-MAPK did not change (Fig. 4B, $\mathrm{P}<0.01$ ). HuMSCs treatment alleviated these changes in DCM rats. Protein expression of p-ERK1/2; however, not p-p38-MAPK was attenuated significantly in the HuMSCs treated DCM rats compared with vehicle-treated 

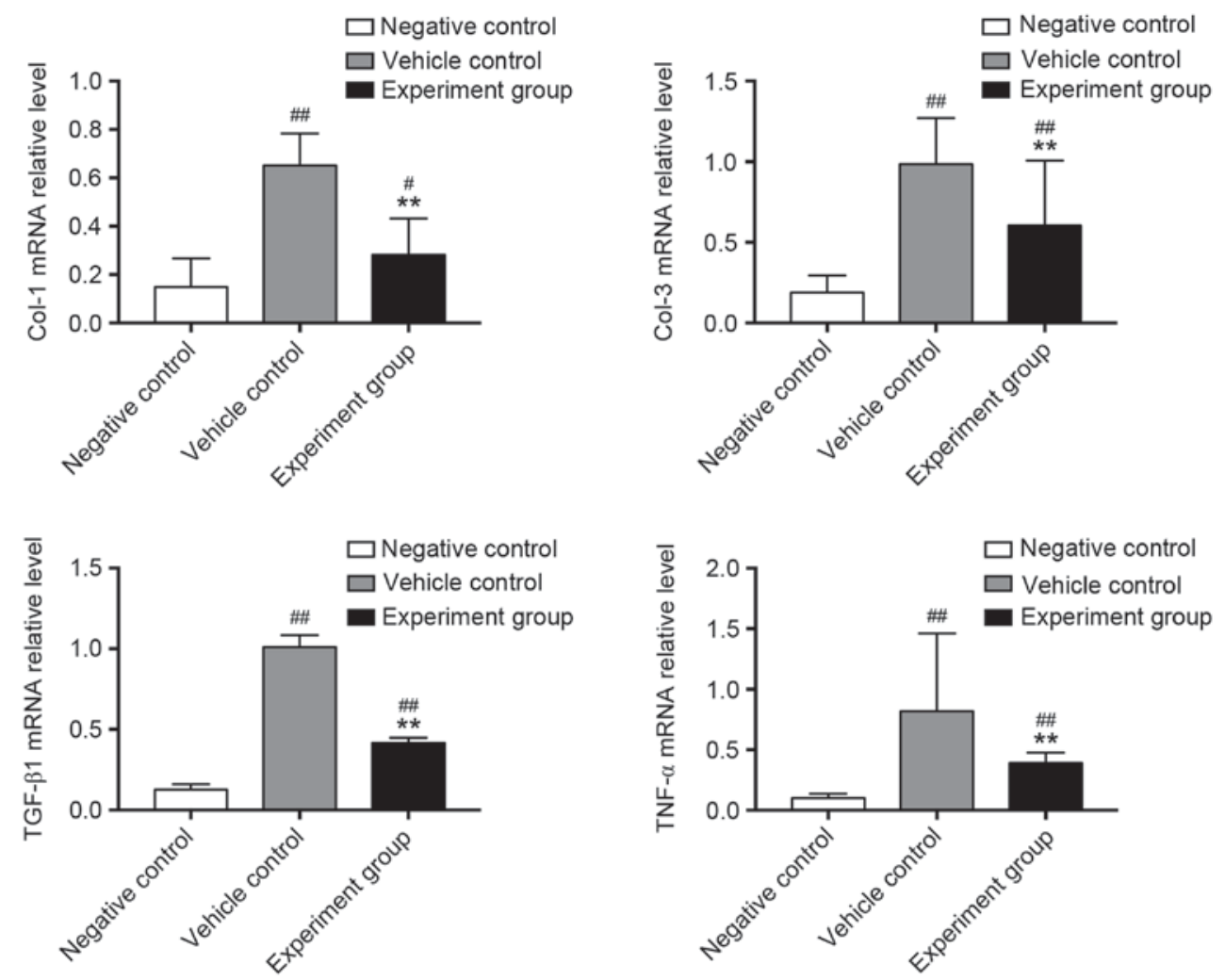

Figure 2. Analysis of myocardial mRNA levels of the extracellular matrix components collagen I and III, and the profibrotic factors TGF- $\beta 1$ and TNF- $\alpha$ by reverse transcription-quantitative polymerase chain reaction of tissues from negative control, vehicle control, and experiment group rats. Relative expression levels are presented. GAPDH was used as the internal standard. ${ }^{\prime \prime} \mathrm{P}<0.05,{ }^{\# \#} \mathrm{P}<0.01$ vs. negative control. ${ }^{*} \mathrm{P}<0.05 ;{ }^{* *} \mathrm{P}<0.01$ vs. vehicle control, $\mathrm{n}=8$. TGF- $\beta 1$, transforming growth factor- $\beta 1$; TNF- $\alpha$, tumor necrosis factor- $\alpha$.

A

Negative control Vehicle control Experiment group

Collagen III

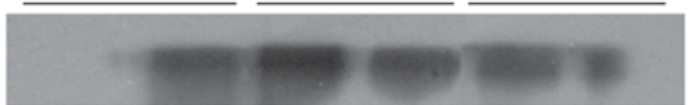

CTGF

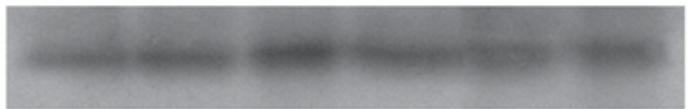

GAPDH
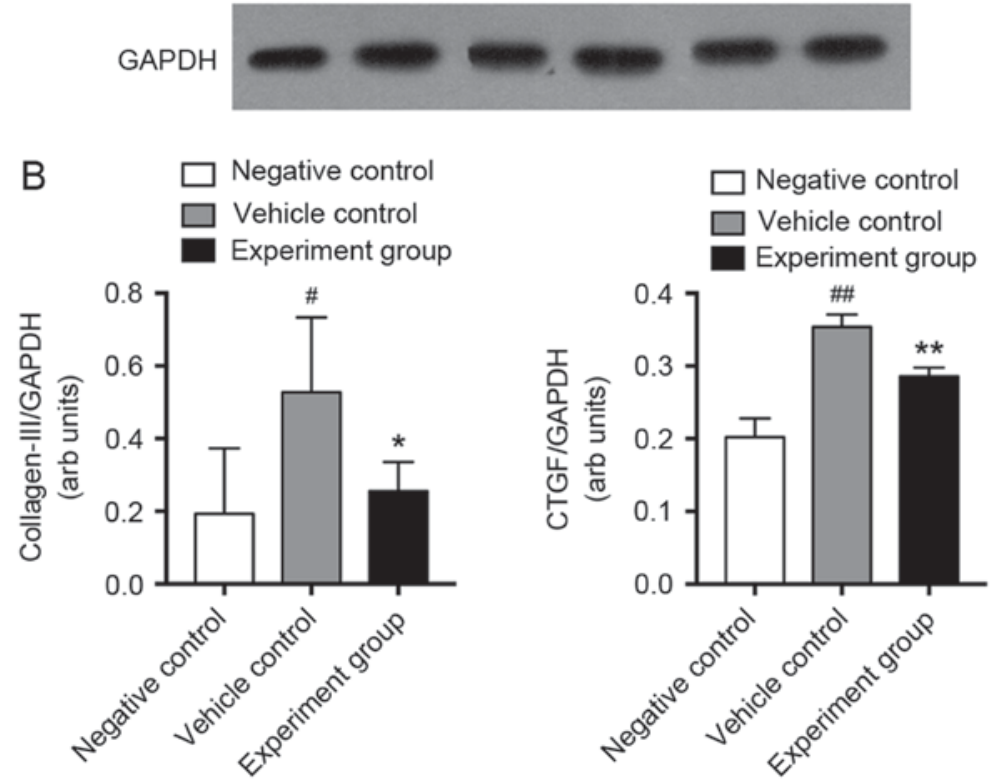

Figure 3. Western blot analysis of CTGF and collagen III expression in negative control, vehicle control, and experimental rats. (A) Western blot analysis of immunolabeled bands for CTGF and collagen III, with GAPDH as an internal control. (B) Ratio of the mean density values of CTGF and Col III relative to GAPDH. ${ }^{\# P}<0.05,{ }^{\# \#} \mathrm{P}<0.01$ vs. negative control; ${ }^{\mathrm{P}}<0.05,{ }^{* *} \mathrm{P}<0.01$ vs. vehicle control, $\mathrm{n}=8$. CTGF, connective tissue growth factor. 

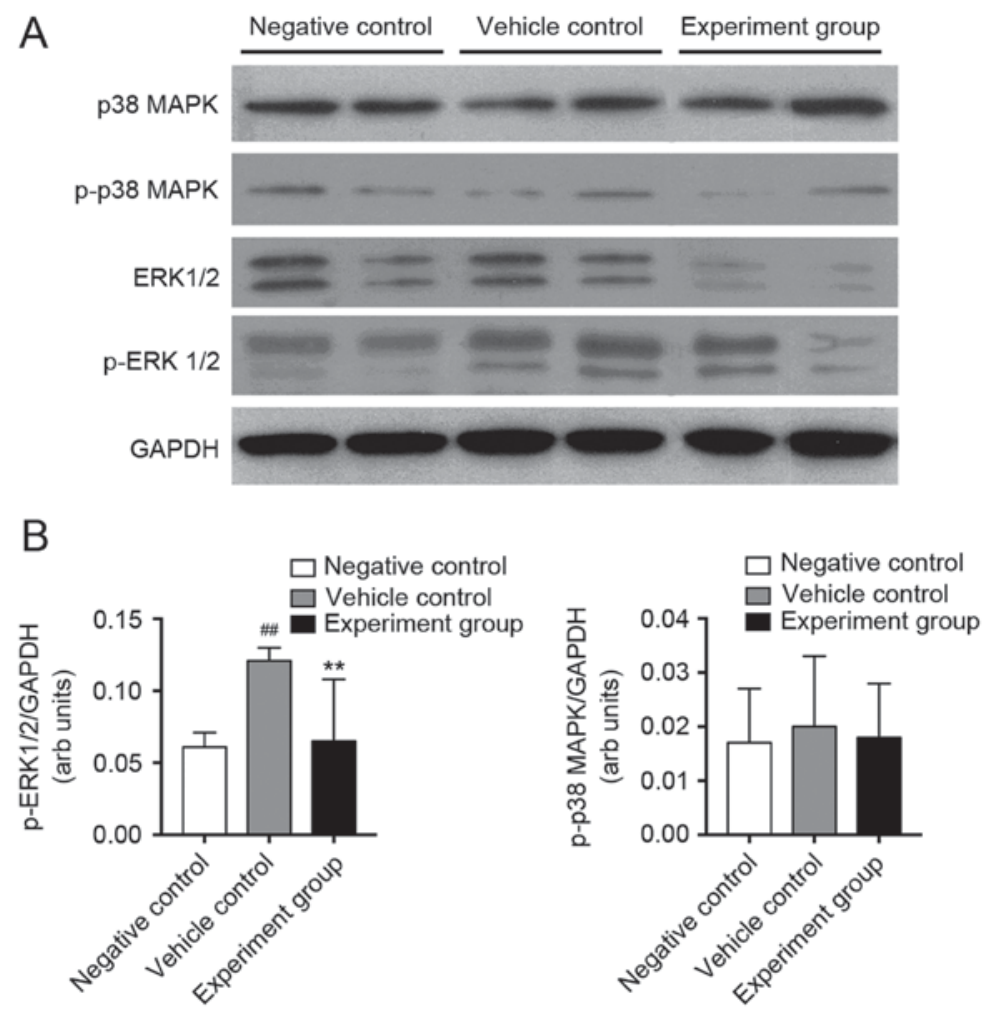

Figure 4. Western blot analysis of p38 MAPK and ERK1/2 phosphorylation in negative control, vehicle control, and experimental rats. (A) Representative western blotting showing immunolabeled bands for p38-MAPK, p-p38 MAPK, ERK1/2, p-ERK1/2, and GAPDH was used as an internal control. (B) Mean density values of p-ERK1/2 and p-p38 MAPK expressed as the ratio relative to GAPDH expression. GAPDH used as an internal control. ${ }^{\# /} \mathrm{P}<0.01 \mathrm{vs.} \mathrm{negative}$ control; * ${ }^{* *}<0.01$ vs. vehicle control, $\mathrm{n}=8$. p38 MAPK, p38 mitogen-activated protein kinase; p-p38 MAPK, phosphorylated-p38 MAPK; ERK1/2, extracellular-signal regulated kinase 1/2; p-ERK1/2, phosphorylated-ERK1/2.

DCM rats (Fig. 4B, P<0.01), indicating that HuMSCs may alter myocardial fibrosis via TNF- $\alpha$, TGF- $\beta$ and ERK1/2 activation.

\section{Discussion}

In the present study, reduced cardiac function in a DCM rat model was associated with LV remodeling, as well as increased myocardial collagen deposition and upregulated myocardial type I and III collagen expression. Treatment with HuMSCs significantly improved LV remodeling, LV systolic function and reduced collagen deposition, likely by inhibiting the TGF- $\beta /$ TNF- $\alpha$, ERK $1 / 2$ signaling pathways.

A loss of cardiomyocytes and an increase in interstitial fibrosis is characteristic for DCM (27). The degree of cardiac fibrosis, leading to passive ventricular stiffness and reduced cardiac function, may be determined by measuring the myocardial collagen volume fraction. Masson's staining revealed a significant increase in collagen deposition in DCM rats. The abnormal deposition of collagen within myocardial tissues was significantly reduced following treatment with HuMSCs. Cardiac fibroblasts, the cells that form the interstitial tissue within the healthy myocardium, are considered to be the major source of collagen and fibrosis after cardiac injury (28). The major fibrillar collagens of the adult heart are type I and III collagens $(29,30)$ and if the level of collagen within the myocardium increases, ventricular compliance may be reduced. The data of the present study indicated that type I and III collagen was upregulated in myocardial tissues of DCM rats; however, their expression levels were significantly reduced following treatment with HuMSCs.

CTGF is another fibrosis-associated molecule that may induce fibrocyte differentiation into a myofibroblast phenotype and increase ECM deposition in the myocardium (31). The data of the present study indicated that CTGF was upregulated in myocardial tissues of DCM rats; however, the expression level was significantly reduced following treatment with HuMSCs.

A previous study determined that TGF- $\beta$ is upregulated in several models of myocardial infarction and DCM (32). It has been previously suggested that TGF- $\beta 1$ is a master switch for inducing myocardial fibrosis and may upregulate the expression of procollagen genes to promote synthesis of ECM components, subsequently leading to fibrosis (32). TGF- $\beta 1$-overexpressing mice had significant cardiac hypertrophy along with interstitial fibrosis (33). TGF- $\beta 1$ levels in dilated and infarcted hearts were reduced following treatment with angiotensin-converting enzyme inhibitors or angiotensin II receptor blockers $(34,35)$. A previous study suggested that the antifibrotic mechanism of HuMSCs in lung fibrogenesis may be associated with TGF- $\beta 1$ downregulation, which may lead to suppression of fibrosis following lung injury (36). The present study determined that HuMSCs downregulated myocardial TGF- $\beta 1$ mRNA expression and led to the suppression of myocardial fibrosis in DCM rats.

TGF- $\beta 1$ activates various non-canonical signaling pathways, including the MAPK and phosphatidylinositol-3 
kinase/Akt pathways $(37,38)$. The progression of FasL-induced DCM and congestive heart failure was prevented by blocking the ERK1/2 pathway, with reductions in fibrosis, inflammation and apoptosis (39). Isoproterenol and cAMP signaling attenuated the profibrotic effect of TGF- $\beta 1$ in cardiac fibroblasts by suppressing ERK1/2 phosphorylation. Additionally, the MEK/ERK pathway is involved in interleukin-17-mediated cardiac fibrosis in EAM (40). In the present study, HuMSC administration significantly reduced ERK1/2 phosphorylation in DCM rats. However, phosphorylation of p38-MAPK was not altered in the three experimental groups. These findings support previous observations $(37,38)$, that the TGF- $\beta /$ ERK $1 / 2$ pathway is an important mediator in cardiac fibrosis. The data of the present study revealed that ERK1/2, not p38-MAPK signaling, was involved with fibrosis in DCM and identified that HuMSCs treatment may effectively protect against cardiac fibrosis by attenuating the activation of the ERK1/2 pathway.

TNF- $\alpha$, a proinflammatory cytokine with a wide range of biological effects, is involved in the pathophysiology of various cardiovascular diseases $(41,42)$. TNF- $\alpha$ overexpression in transgenic mice may lead to adverse cardiac remodeling, characterized by increased total matrix metalloproteinase (MMP) activity and increased fibrosis (43). Inhibition of TNF- $\alpha$ may protect from myocardial inflammation and fibrosis through reduced ERK phosphorylation in experimental diabetic cardiomyopathy (44). In the present study, administration of HuMSCs effectively reduced myocardial mRNA expression of TNF- $\alpha$ in DCM rats. Therefore, it is possible that similar to the TGF- $\beta /$ ERK1/2 pathway, HuMSCs alter TNF- $\alpha /$ ERK1/2 signaling and may alleviate cardiac fibrosis, which subsequently improves ventricular compliance and cardiac function.

In conclusion, the present findings demonstrated that injected HuMSCs improve cardiac function by attenuating myocardial collagen network remodeling via downregulation of TGF- $\beta 1$ and TNF- $\alpha$ expression and activation of ERK1/2 signaling in DCM rats. Therefore, HuMSC treatment may be a potential therapeutic avenue for treatment of DCM.

\section{Acknowledgements}

The present study was supported by grants from the National Natural Science Foundation of China (grant no. 81671525 and 81070478), the Department of Health of Guangdong Province (grant no. B2013276), Medical Science and Technology Research Foundation of Guangdong Province (grant no. A2015247), the Science and Technology Program Project of Shantou (grant nos. 2012165 and 2013), the Basic and Clinical Scientific Research Foundation of Shantou University Medical College (grant no. 201410), the Science and Technology Program of Shenzhen (grant no. JCYJ20150402092905162) and the Research Project of Health and Family Planning Commission of Shenzhen Municipality (grant no. 201501053).

\section{References}

1. Krejci J, Mlejnek D, Sochorova D and Nemec P: Inflammatory Cardiomyopathy: A current view on the pathophysiology, diagnosis, and treatment. Biomed Res Int 2016: 4087632, 2016.

2. Lauer B, Schannwell M, Kühl U, Strauer BE and Schultheiss HP: Antimyosin autoantibodies are associated with deterioration of systolic and diastolic left ventricular function in patients with chronic myocarditis. J Am Coll Cardiol 35: 11-18, 2000.
3. Binah O: Pharmacologic modulation of the immune interaction between cytotoxic lymphocytes and ventricular myocytes. J Cardiovasc Pharmacol 38: 298-316, 2001.

4. Kodama M, Matsumoto Y, Fujiwara M, Masani F, Izumi T and Shibata A: A novel experimental model of giant cell myocarditis induced in rats by immunization with cardiac myosin fraction. Clin Immunol Immunopathol 57: 250-262, 1990.

5. Spezzacatene A, Sinagra G, Merlo M, Barbati G, Graw SL, Brun F, Slavov D, Di Lenarda A, Salcedo EE, Towbin JA, et al: Arrhythmogenic phenotype in dilated cardiomyopathy: Natural history and predictors of life-threatening arrhythmias. J Am Heart Assoc 4: e002149, 2015.

6. Williams AR and Hare JM: Mesenchymal stem cells: Biology, pathophysiology, translational findings, and therapeutic implications for cardiac disease. Circ Res 109: 923-940, 2011.

7. Sanganalmath SK and Bolli R: Cell therapy for heart failure: A comprehensive overview of experimental and clinical studies, current challenges, and future directions. Circ Res 113: 810-834, 2013.

8. Kelkar AA, Butler J, Schelbert EB, Greene SJ, Quyyumi AA, Bonow RO, Cohen I, Gheorghiade M, Lipinski MJ, Sun W, et al: Mechanisms contributing to the progression of ischemic and nonischemic dilated cardiomyopathy: Possible modulating effects of paracrine activities of stem cells. J Am Coll Cardiol 66: 2038-2047, 2015.

9. Kizilay Mancini O, Shum-Tim D, Stochaj U, Correa JA and Colmegna I: Age, atherosclerosis and type 2 diabetes reduce human mesenchymal stromal cell-mediated T-cell suppression. Stem Cell Res Ther 6: 140, 2015.

10. Hare JM, Fishman JE, Gerstenblith G, Zambrano JP, Suncion VY, Tracy M, Ghersin E, Johnston PV, Brinker JA, et al: Comparison of allogeneic vs. autologous bone marrow-derived mesenchymal stem cells delivered by transendocardial injection in patients with ischemic cardiomyopathy: The POSEIDON randomized trial. Jama 308: 2369-2379, 2012.

11. Karantalis V and Hare JM: Use of mesenchymal stem cells for therapy of cardiac disease. Circ Res 116: 1413-1430, 2015.

12. Golpanian S, Wolf A, Hatzistergos KE and Hare JM: Rebuilding the damaged heart: Mesenchymal stem cells, cell-based therapy, and engineered heart tissue. Physiol Rev 96: 1127-1168, 2016.

13. Wang HS, Hung SC, Peng ST, Huang CC, Wei HM, Guo YJ, Fu YS, Lai MC and Chen CC: Mesenchymal stem cells in the Wharton's jelly of the human umbilical cord. Stem Cells 22: 1330-1337, 2004.

14. Cho PS, Messina DJ, Hirsh EL, Chi N, Goldman SN, Lo DP, Harris IR, Popma SH, Sachs DH and Huang CA: Immunogenicity of umbilical cord tissue derived cells. Blood 111: 430-438, 2008.

15. Batsali AK, Kastrinaki MC, Papadaki HA and Pontikoglou C: Mesenchymal stem cells derived from Wharton's Jelly of the umbilical cord: Biological properties and emerging clinical applications. Curr Stem Cell Res Ther 8: 144-155, 2013.

16. Sun L, Wang D, Liang J, Zhang H, Feng X, Wang H, Hua B, Liu B, Ye S, Hu X, et al: Umbilical cord mesenchymal stem cell transplantation in severe and refractory systemic lupus erythematosus. Arthritis Rheum 62: 2467-2475, 2010.

17. Wang L, Wang L, Cong X, Liu G, Zhou J, Bai B, Li Y, Bai W, Li M, Ji H, et al: Human umbilical cord mesenchymal stem cell therapy for patients with active rheumatoid arthritis: Safety and efficacy. Stem Cells Dev 22: 3192-3202, 2013.

18. Wang H, Qiu X, Ni P, Qiu X, Lin X, Wu W, Xie L, Lin L, Min J, Lai X, et al: Immunological characteristics of human umbilical cord mesenchymal stem cells and the therapeutic effects of their transplantion on hyperglycemia in diabetic rats. Int $\mathrm{J}$ Mol Med 33: 263-270, 2014.

19. Can A, Ulus AT, Cinar O, Topal Celikkan F, Simsek E, Akyol M, Canpolat U, Erturk M, Kara F and Ilhan O: Human umbilical cord mesenchymal stromal cell transplantation in myocardial ischemia (HUC-HEART Trial). A study protocol of a Phase 1/2, controlled and randomized trial in combination with coronary artery bypass grafting. Stem Cell Rev 11: 752-760, 2015.

20. Liu CB, Huang H, Sun P, Ma SZ, Liu AH, Xue J, Fu JH, Liang YQ, Liu B, Wu DY, et al: Human umbilical cord-derived mesenchymal stromal cells improve left ventricular function, perfusion, and remodeling in a porcine model of chronic myocardial ischemia. Stem Cells Transl Med 5: 1004-1013, 2016.

21. Cheng M, Wu G, Song Y, Wang L, Tu L, Zhang L and Zhang C: Celastrol-induced suppression of the MiR-21/ERK signalling pathway attenuates cardiac fibrosis and dysfunction. Cell Physiol Biochem 38: 1928-1938, 2016. 
22. Wu H, Li GN, Xie J, Li R, Chen QH, Chen JZ, Wei ZH, Kang LN and $\mathrm{Xu}$ B: Resveratrol ameliorates myocardial fibrosis by inhibiting ROS/ERK/TGF- $\beta$ /periostin pathway in STZ-induced diabetic mice. BMC Cardiovasc Disord 16: 5, 2016.

23. Soetikno V, Sari FR, Sukumaran V, Lakshmanan AP, Mito S, Harima M, Thandavarayan RA, Suzuki K, Nagata M, Takagi R and Watanabe $\mathrm{K}$ : Curcumin prevents diabetic cardiomyopathy in streptozotocin-induced diabetic Rats: Possible involvement of PKC-MAPK signaling pathway. Eur J Pharm Sci 47: 604-614, 2012.

24. Wang HW, Lin LM, He HY, You F, Li WZ, Huang TH, Ma GX and Ma L: Human umbilical cord mesenchymal stem cells derived from Wharton's jelly differentiate into insulin-producing cells in vitro. Chinese Med J (Engl) 124: 1534-1539, 2011.

25. Zhang C, Zhou G, Cai C, Li J, Chen F, Xie L, Wang W, Zhang Y, Lai X and Ma L: Human umbilical cord mesenchymal stem cells alleviate acute myocarditis by modulating endoplasmic reticulum stress and extracellular signal regulated 1/2-mediated apoptosis. Mol Med Rep 15: 3515-3520, 2017.

26. Livak KJ and Schmittgen TD: Analysis of relative gene expression data using real-time quantitative PCR and the 2(-Delta Delta C(T)) method. Methods 25: 402-408, 2001.

27. Dec GW and Fuster V: Idiopathic dilated cardiomyopathy. N Engl J Med 331: 1564-1575, 1994.

28. Kania G, Blyszczuk P and Eriksson U: Mechanisms of cardiac fibrosis in inflammatory heart disease. Trends Cardiovasc Med 19: 247-252, 2009.

29. Eckhouse SR and Spinale FG: Changes in the myocardial interstitium and contribution to the progression of heart failure. Heart Fail Clin 8: 7-20, 2012.

30. Broberg CS and Burchill LJ: Myocardial factor revisited: The importance of myocardial fibrosis in adults with congenital heart disease. Int J Cardiol 189: 204-210, 2015.

31. Rosin NL, Falkenham A, Sopel MJ, Lee TD and Legare JF: Regulation and role of connective tissue growth factor in AngII-induced myocardial fibrosis. Am J Pathol 182: 714-726, 2013.

32. Dobaczewski M, Chen W and Frangogiannis NG: Transforming growth factor (TGF)- $\beta$ signaling in cardiac remodeling. J Mol Cell Cardiol 51: 600-606, 2011.

33. Rosenkranz S, Flesch M, Amann K, Haeuseler C, Kilter H, Seeland U, Schlüter KD and Böhm M: Alterations of beta-adrenergic signaling and cardiac hypertrophy in transgenic mice overexpressing TGF-beta(1). Am J Physiol Heart Circ Physiol 283: H1253-H1262, 2002.

34. Sukumaran V, Watanabe K, Veeraveedu PT, Thandavarayan RA, Gurusamy N, Ma M, Yamaguchi K, Suzuki K, Kodama M and Aizawa Y: Telmisartan, an angiotensin-II receptor blocker ameliorates cardiac remodeling in rats with dilated cardiomyopathy. Hypertension Res 33: 695-702, 2010.
35. Yu CM, Tipoe GL, Wing-Hon Lai K and Lau CP: Effects of combination of angiotensin-converting enzyme inhibitor and angiotensin receptor antagonist on inflammatory cellular infiltration and myocardial interstitial fibrosis after acute myocardial infarction. J Am Coll Cardiol 38: 1207-1215, 2001.

36. Moodley Y, Atienza D, Manuelpillai U, Samuel CS, Tchongue J, Ilancheran S, Boyd R and Trounson A: Human umbilical cord mesenchymal stem cells reduce fibrosis of bleomycin-induced lung injury. Am J Pathol 175: 303-313, 2009.

37. Massague J: Integration of Smad and MAPK pathways: A link and a linker revisited. Genes Dev 17: 2993-2997, 2003

38. Guo $X$ and Wang XF: Signaling cross-talk between TGF-beta/BMP and other pathways. Cell research 19: 71-88, 2009.

39. Huby AC, Turdi S, James J, Towbin JA and Purevjav E: FasL expression in cardiomyocytes activates the ERK1/2 pathway, leading to dilated cardiomyopathy and advanced heart failure. Clin Sci (Lond). 130: 289-299, 2016.

40. Liu Y, Zhu H, Su Z, Sun C, Yin J, Yuan H, Sandoghchian S, Jiao Z, Wang $S$ and Xu H: IL-17 contributes to cardiac fibrosis following experimental autoimmune myocarditis by a PKC $\beta /$ Erk1/2/NF-kappaB-dependent signaling pathway. Int Immunol 24: 605-612, 2012.

41. Sriramula S, Haque M, Majid DS and Francis J: Involvement of tumor necrosis factor-alpha in angiotensin II-mediated effects on salt appetite, hypertension, and cardiac hypertrophy. Hypertension 51: 1345-1351, 2008.

42. Sun M, Chen M, Dawood F, Zurawska U, Li JY, Parker T, Kassiri Z, Kirshenbaum LA, Arnold M, Khokha R and Liu PP: Tumor necrosis factor-alpha mediates cardiac remodeling and ventricular dysfunction after pressure overload state. Circulation 115: 1398-1407, 2007.

43. Sivasubramanian N, Coker ML, Kurrelmeyer KM, MacLellan WR, DeMayo FJ, Spinale FG and Mann DL: Left ventricular remodeling in transgenic mice with cardiac restricted overexpression of tumor necrosis factor. Circulation 104: 826-831, 2001.

44. Westermann D, Van Linthout S, Dhayat S, Dhayat N, Schmidt A Noutsias M, Song XY, Spillmann F, Riad A, Schultheiss HP and Tschöpe C: Tumor necrosis factor-alpha antagonism protects from myocardial inflammation and fibrosis in experimental diabetic cardiomyopathy. Basic Res Cardiol 102: 500-507, 2007.

This work is licensed under a Creative Commons Attribution-NonCommercial-NoDerivatives 4.0 International (CC BY-NC-ND 4.0) License. 